Mostafa Alimehr ${ }^{1}$, Samira Malayen², Fereshteh Sohrabi Vafa ${ }^{3}$, Mohsen Jalili Tahmasebi ${ }^{4}$, Maryam Nikbina ${ }^{5}$, Kobra Doostifar ${ }^{6}$

${ }^{1}$ Department of Health Services Management, Dezful University of Medical Sciences, Dezful, Iran

2Department of Midwifery, Dezful University of Medical Sciences, Dezful, Iran

${ }^{3}$ Department of Community Medicine, School of Medicine, Dezful University of Medical Sciences, Dezful, Iran

${ }^{4}$ Department of Health Services Management, Shahrekord University of Medical Sciences, Shahrekord, Iran

${ }^{5}$ Department of Nursing and Midwifery, Shoushtar Faculty of Medical Sciences, Shoushtar, Iran

${ }^{6}$ Department of Public Health, Shoushtar Faculty of Medical Sciences, Shoushtar, Iran

\title{
The impact of coronavirus disease (COVID-19) on quality of life in diabetic patients
}

\section{ABSTRACT}

Background. Diabetes is one of the most prevalent metabolic diseases and it includes disabling complications and chronic and invisible progression. On the one hand, this disease imposes high financial expenses on the patient and, on the other hand, reduces the quality of life. Presently, the COVID-19 epidemic has been an international concern, and evidence implies that people who have the underlying disease are at higher risk for the virus and will experience serious problems if they develop the disease.

Methods. This research is a qualitative study with a kind of phenomenological investigation that has been conducted to investigate the patient's experiences and personal feelings with diabetes in terms of the effects of corona disease on their quality of life. In this study, the semi-structured interview method was utilized to collect information. The number of sample included 15 patients referred to health centers, and each interview was recorded with the patient's consent and was written on paper and analyzed.

Results. The data of this study were classified into 6 main themes and 19 sub-themes after analysis. The main themes of the information obtained from this study included knowledge and awareness, psychological effects, entertainment, and recreational programs,

Address for correspondence:

Samira Malayen

Department of Midwifery

Dezful University of Medical Sciences

Dezful, Iran

e-mail: s.malayen2021@gmail.com

Clinical Diabetology 2021, 10; 3: 237-242

DOI: 10.5603/DK.a2021.0024

Received: 13.01.2021

Accepted: 29.01 .2021 patient's family, medical care, and communication with patients placing the greatest emphasis on medical care. Stress caused by corona disease and depression were the most important psychological effects expressed by diabetic patients. They also expressed some serious medical care problems, including the reduction of referrals to health centers for follow-up and care and the shortage of physicians and health personnel to provide services.

Conclusion. This study suggests that diabetic patients experience many different problems due to corona disease. These problems influence the diabetic patients' quality of life, so we need to understand better and solve the diabetic patients' problems, make more effort, and perform more suitable planning to improve their health and quality of life. (Clin Diabetol 2021; 10; 3: 237-242)

Key words: COVID-19, type 2 diabetes mellitus, quality of life

\section{Introduction}

Chronic diseases affect more than 35 million patients annually and are responsible for_nearly two-thirds of all deaths around the world [1]. According to the World Health Organization, $60 \%$ of the causes of death and $43 \%$ of the global burden of disease are related to non-communicable diseases and diabetes is one of the most common chronic diseases around the world [2]. Diabetes will be the seventh leading cause of death in the world by 2030 and more than $80 \%$ of deaths caused by it will occur in low- and middle-income countries [3].

Type 2 diabetes remarkably affects patients' quality of life due to chronic disease, disability, and the 
necessity for lifelong patient care [4]; these disorders, which appear in various aspects of a person's life with diabetes, due to the impossibility of complete recovery result in negative effects on the patient's quality of life. Hence, one of the principal purposes in the medical care of this type of patients is to improve their quality of life [5]. Accordingly, the quality-of-life index is one of the significant health consequences that are employed for purposes such as measuring the influence of health services [6]. In a study entitled "Quality of Life for Type 2 Diabetes in a Hospital in Thailand", conducted by Shankawi et al. in 2020, it was specified that diabetes influences all aspects of a patient's life and causes mental disorders and even depression in some patients [7]. Chronic complications of diabetes reduce the patient's physical and mental health and reduce their quality of life. It is extremely important to identify the problems of these patients and attempt to solve them due to its high costs for the individual and society [8]. On the other hand, the concern of diabetic patients was increased with the outbreak of an unexplored and new disease called COVID-19 in the world, the first case of which was reported in Wuhan, China, in December 2019 [9]. Although different measures were taken to control the spread of the disease in the country, the rate of transmission of the virus was very high; hence, the World Health Organization declared COVID-19 as a pandemic disease [10]. The disease is more prevalent in the elderly and those with underlying disease, and results in death. Hence, health policymakers have implemented various measures to control the spread of the virus by giving priority to the most vulnerable people in society [11]. Malek et al., in a study entitled "Managing Diabetes during the COVID-19 Epidemic in 2020: A Statement of Iranian Expert Opinion", indicated that Case Fatality Rate (CFR) in people with diabetes was $9.2 \%$, while in healthy people with no specific disease it was $1.4 \%$, and because diabetes is a common disease in the world, health care professionals are very concerned about the comprehensive development of acute COVID-19 respiratory syndrome and its influence on patients with diabetes [12]. Accordingly, because diabetes is one of the priorities among chronic diseases, it has a particular place in the epidemic situations of COVID-19 crisis and diabetic patients require more attention from family members and health care personnel, so this study was conducted to describe the impact of COVID-19 on diabetic patients' quality of life in Andimeshk, Iran.

\section{Methods}

This study was a qualitative study performed in 2020-2021. The aim of the study was to evaluate the health care system. It was a kind of phenomenological study, which was conducted in Andimeshk, Iran. The statistical population included diabetic patients who were referred to health centers in the city to receive services. Accordingly, after obtaining permission from the relevant bodies, we referred to health centers in the city, and 8 centers were selected by cluster sampling among the health centers in Andimeshk. Then, people who satisfied the inclusion criteria were selected by examining the list of cases of diabetic patients who were treated in health centers. Later, individuals were listed according to the table of random numbers, and they were invited to participate in the study. If they did not desire to, they were replaced by other randomly selected eligible patients.

This study's sample size was similar to that of qualitative studies to achieve information saturation, and where no new data were obtained, sampling was completed. In this study, the number of interviewees was 15 (9 were female and 6 were male). The sampling criterion was having information about diabetes and COVID-19. The interviews were semi-structured and in-depth and were conducted by setting a prior appointment and selecting a location from the participants' perspective. Each interview lasted from 30 to 40 minutes, according to the participants' conditions and requests. Each interview began with an open-ended question so that the respondent could feel relaxed and openly express his/her feelings, thoughts, and opinions.

Two interviews were conducted with people who were not selected as participants to determine the validity and ensure that the questions have meaning to the respondents. According to their prospects, the needed corrections were made to solve the shortcomings. Also, the people who participated in the meeting were asked for permission to record the interview prior to the meeting, and due to the lack of objections to this issue, all interviews were recorded, and the recorded interviews were transcribed immediately after the interviews, and then analyzed. These data were employed as a guide in the later stages of the work. The interviews were examined and analyzed using thematic analysis. Accordingly, two researchers read the results of the interviews several times and divided them into semantic units. The semantic units were then summarized, and the units that had equal meanings were placed under a code title. Consequently, the codes were classified, and the resulting meaning was extracted as the principal theme. In cases where there was dissimilarity among researchers, doubts were resolved through discussion, and an agreement was achieved. In this study, ethical principles, including confidentiality of the information and obtaining informed consent from the participants for interviewing and recording the interview, were ob- 
served while preserving anonymity and confidentiality. The right to leave the research at any time was one of the ethical considerations that was considered and observed in this study.

\section{Results}

Theme 1. Knowledge and information

Knowledge and information play a determining role in COVID-19 and diabetic patients. Information about this disease can better understand how to prevent and control the disease and suffer less from its complications. This main theme involves three sub-axes:

- Medical information concerning diabetes and COVID-19;

- Awareness concerning observing health protocols;

- Awareness concerning daily news about COVID-19.

Several interviewees commented on this regard:

"According to the advice of health experts and specialists who are invited to television programs, our health information on the prevention of COVID-19 has expanded" (interviewee 3), and another one said: "Our knowledge concerning observing health protocols according to the media and also warning posters "Installed in public offices and places have been increased «" (interviewee 10). Another interviewee said: "We become informed of the statistics and information of COVID-19 by following up-to-date news from prominent media and cyberspace" (interviewee 9).

\section{Theme 2. Mental}

Fear and anxiety are very common in such crises; hence, this fear and anxiety when the disease threatens the person's life or when it is possible that relatives and loved ones may be infected with the virus can have increasingly negative effects on the people's psyche, particularly patients who have chronic diseases, especially diabetes. This main theme also involves four sub-axes:

- Stress and anxiety;

- Depression;

- Aggression;

- Boredom.

The interviewees stated in this regard:

"I'm constantly stressed that I'm not getting COVID-19 disease or that I' $m$ thinking I'm going to die if I get it" (interviewee 2); another one said: "We have heard about the death of many people or from relatives and friends who have died because of this disease and we have become depressed" (interviewee 6). Another interviewee said: "I have become aggressive with my family members because I have been at home for a long time and I do not go out of home and think of this disease" (interviewee 7).

\section{Theme 3. Recreational and entertainment} programs

One of the requirements of humans is the need for healthy recreation and travel, and that recreational activities and going to recreational places have great effects on people's quality of life, particularly people with chronic diseases.

This main theme also involves three sub-axes:

- Reducing sports activities;

- Reducing travel;

- Reducing the presence in recreational and wellbeing places.

"I always went to the gym to exercise to control my diabetes, but despite this disease, my exercise is limited and I exercise at home" (interviewee 9) and another one said: "We used to travel with family and friends several times a year to have a spirit, but due to the outbreak of the disease, we stayed at home and did not travel at all" (interviewee 13). Another interviewee said: "We used to go to parks and amusement parks and spent our time, but since the disease has been spread, we have canceled all programs" (interviewee15).

\section{Theme 4. Patient's family}

Diabetes imposes a lot of psychological, social, and economic difficulty on families. Additionally, the family environment can play an extraordinary role in adapting diabetic patients to lifestyle changes to control blood sugar and prevent its complications correctly. It can be stated that diabetic patients that are supported by their family will be able to adapt more to COVID-19 and will have longer survival compared to patients without family support, but the effects of chronic disease not only obstruct patients' lives but also affects caregivers.

This main theme also covers 3 sub-axes as follows:

- Caring for patients at home;

- Providing the patient's medicine from health centers and pharmacies;

- Anxiety caused by thinking about the patient's condition.

Some interviewees commented on this regard:

"Family members should be careful about my diet and medication" (interviewee14). Another one said: "Family members purchase my medication from the pharmacy and do not allow me to go out because of COVID-19" (interviewee 8) and another interviewee said "The anxiety caused by my family thinking about my diabetes with COVID-19 makes them very worried" (interviewee 9). 


\section{Theme 5. Health care}

Diabetes is related to high levels of health care resources and costs. Diabetic patients require extensive health services and access to medical treatment and care services, and on the other hand, these patients have many problems in terms of disease management and high costs of the disease.

This main theme also covers 3 sub-axes as follows:

- Reducing referrals to centers for follow-up and care;

- Lack of personnel and physicians to provide services for telemedicine visits;

- Problems related to receiving insulin. Some interviewees commented on this regard:

"Despite having COVID-19, I show up less often to health centers for follow-up and care" (interviewee 5) and another interviewee said: "One time I arrived to the health center and observed that the workload of the personnel was high due to COVID-19 and half of the personnel worked in a telecommuting way" (interviewee 4). "We had a shortage of insulin in the COVID-19 crisis", said another interviewee (interviewee 3).

\section{Theme 6. Communications}

The policy of creating distance by restraining interactions in the social dimension and in the individual dimension to control and limit the spread of the COVID-19 is precisely the position that a therapeutic problem deals with the social problem. In other words, currently, the most efficient way to cope with the spread of the virus is to slow down the wheels of social life to reduce the burden of disease, and the health care system does not meet the risk of collapse.

This main theme also covers three sub-axes as follows:

- Reducing communication with relatives and friends;

- Reducing presence in society to do personal work;

- Changing lifestyle.

Some interviewees commented on this respect:

"Communication with relatives and friends was cut off with the outbreak of this disease" (interviewee 15), and another one said that this disease has caused us to appear less in society to defeat the disease (interviewee 14). Another interviewee also announced that his lifestyle has changed because of this disease (interviewee 6).

\section{Discussion}

The results achieved by this study indicate the influence of COVID-19 on diabetes in different dimensions; the most important priorities from the interviewees' perspective were the issues of knowledge and aware- ness about COVID-19, the psychological effect of the disease, well-being and recreation programs, family care, medical care, and communication (Table 1).

According to the findings achieved by interviews with experts, the knowledge and information concerning diabetic patients are very helpful in preventing COVID-19. Kumar Singh et al. showed in a study conducted in 2020 entitled "COVID-19: Assessing Knowledge and Information in Indian Society" people were well aware of the prevalence of COVID-19 and its preventive measures; $98 \%$ of respondents answered that the virus was transmitted from person to person, and 95 responded that a virus has caused the disease. People understand the importance of social distance and other preventive measures appointed by the government in good faith for the COVID-19 and follow credible sources and media to receive information about the COVID-19, the virus and common symptoms, and they were informed of its prevention [13].

The psychological damages of COVID-19 cannot be rejected; the psychological, mental and stress dimensions of this disease are not less than the disease's danger. Anxiety and concern about the family and the fear of being infected with the disease are critical.

A study conducted by Nader Salari et al. [14] in 2020 entitled "Prevalence of stress, anxiety, and depression among the general population during COVID-19 epidemic: A systematic study and meta-analysis" explained that COVID-19 significantly affects general mental health. Accordingly, monitoring the mental health of the population during crises such as COVID-19 is an instant priority. The study aims to analyze research and the findings regarding the prevalence of stress, anxiety, and depression in the general population, particularly in chronic patients during the COVID-19 epidemic [14].

There are limited opportunities for recreation and exercise due to the epidemic of COVID-19; therefore, we should stay at home, and staying at home can create fatigue and burnout for diabetics, resulting in aggression and stress. In this regard, Azter et al. [15], in an article in 2020 entitled "Physical activity during locking due to COVID-19: Recommendations", showed that it is reasonable that limitation due to COVID-19 has decreased the level of physical activity in the general population, and it has also reduced meetings with friends and travel; hence, people should entertain themselves at home with recreational and sports equipment and even reading books [15].

A person's family members, spouse, friends, and colleagues who suffer from diabetes are also affected by diabetes. Fear of the disease, its complications and feelings of helplessness, ambiguity about the future, 
Table 1. Themes and sub-themes related to the impact of COVID-19 on quality of life of diabetic patients

\begin{tabular}{|c|c|}
\hline Main themes & Sub themes \\
\hline \multirow[t]{3}{*}{ Knowledge and information } & 1. Medical information about diabetes and COVID-19 \\
\hline & 2. Information on the observance of health protocols \\
\hline & 3. Information on the daily news about COVID-19 \\
\hline \multirow[t]{4}{*}{ Mental dimension } & 1. Stress and anxiety \\
\hline & 2. Depression \\
\hline & 3. Aggression \\
\hline & 4. Intolerance \\
\hline \multirow[t]{3}{*}{ Recreational and entertainment programs } & 1. Reducing sports activities \\
\hline & 2. Reducing travel \\
\hline & 3. Reducing the presence in recreational and welfare places \\
\hline \multirow[t]{3}{*}{ Patient's family } & 1.The burden of caring for patients at home \\
\hline & 2. Providing the patient's medicine from health centers and pharmacies \\
\hline & 3. Anxiety caused by reflecting on the patient's condition \\
\hline \multirow[t]{3}{*}{ Health care } & 1. Reducing referrals to health centers for follow-up and care \\
\hline & 2. Lack of personnel and physicians to provide telecommunication services \\
\hline & 3. Problems with insulin intake \\
\hline \multirow[t]{3}{*}{ Communication } & 1. Reducing communication with relatives and friends \\
\hline & 2. Reducing presence in society to do personal work \\
\hline & 3. Changing the lifestyle \\
\hline
\end{tabular}

anxiety, anger, economic worries, and stress in interpersonal relationships are factors related to the psychological and social tensions in diabetes; the family as the most fundamental element of society is responsible for proper and appropriate health care for the patient. Irene et al. [16] conducted a study in 2020 entitled "Family care is facing an epidemic crisis", which showed that the COVID-19 epidemic provides unique challenges to the families of patients and those who provide services to them, as well as those who take care of patients at home, and the results of their studies showed that patients, particularly those with chronic diseases require family care more than ever in this epidemic [16].

Diabetics have problems that it is necessary and required to take care of them. If they are left untreated and uncared, they may develop into more acute diseases dominating their lives. Abdi et al. [17] conducted a study in 2020 entitled "Diabetes and COVID-19: A Systematic Review of Evidence", which revealed that an increase in the incidence of diabetes with the prevalence of COVID-19 shows that care for diabetics should be extended to prevent additional complications and reduce the risk of death [17].

Some pervasive social phenomena at the society level, such as the highly contagious COVID-19 epidemic, created the grounds for individual and group restrictions, particularly for people with underlying diseases such as diabetes, and affected highly society, particularly families. Restrictions on commuting, reducing working hours, voluntary or forced home quarantines, the closure of some businesses, and even unemployment caused by the disease have regularly had destructive psychological impacts on society.

Fisher et al. [18] conducted a study in 2020 entitled "Community, work, and family at the time of COVID-19" and showed that COVID-19 has influenced all aspects of people's lifestyles and has brought up confusing situations for society. Also, people with an underlying disease cannot follow up and take care of their disease and have had problems with referring to pharmacies and health centers to obtain their essential medicines [18].

\section{Conclusions}

Considering this point that people with diabetes are among the groups at risk for COVID-19, and the findings have shown that the severity of COVID-19 in people with diabetes is much higher than in healthy people, these people should take more care of their health than others and follow the health recommendations against COVID-19 more carefully than other people._People with diabetes should attempt as much as possible not to endanger themselves to COVID-19 and to avoid commuting around the city and communication with people suspected or infected with the coronavirus. Hence the Ministry of Health should take actions to ensure that people with diabetes are properly cared for and followed-up and to provide them with 
essential medications. Families with diabetic patients should be more cautious in caring for their patients and strive as much as possible to prevent these patients from being present in society; they should also observe health protocols at home.

\section{Acknowledgments}

The authors wish to thank the patients who participated in the study.

\section{Ethics approval}

Ethical approval was obtained from the Shoushtar faculty of Medical Sciences (IR.SHOUSHTAR.REC. 1399.012).

\section{Conflicts of interest}

There are no conflicts of interest.

\section{REFERENCES}

1. Karimi S, Javadi M, Jafarzadeh F. Economic burden and costs of chronic diseases in Iran and the world. Health Inf Manag. 2012; 8(7): 984-996.

2. Jalilian $\mathrm{H}$, Pezeshki MZ, Torkzadeh $\mathrm{L}$, et al. Health care seeking behaviors in type 2 diabetic patients in East Azerbaijan. Clinical Diabetology. 2020; 8(6): 292-302, doi: 10.5603/dk.2019.0031.

3. Mathers $C D$, Loncar D. Projections of global mortality and burden of disease from 2002 to 2030. PLoS Med. 2006; 3(11): e442, doi: 10.1371/journal.pmed.0030442, indexed in Pubmed: 17132052.

4. Moradi A, Alavi SM, Salimi M, et al. The effect of short message service (SMS) on knowledge and preventive behaviors of diabetic foot ulcer in patients with diabetes type 2. Diabetes Metab Syndr. 2019; 13(2): 1255-1260, doi: 10.1016/j.dsx.2019.01.051, indexed in Pubmed: 31336474.

5. Saadatjoo SA, Rezvanee MR, Tabyee SH, et al. Life quality comparison in type 2 diabetic patients and none diabetic persons. Modern Care Journal. 2012; 9(1): 24-31.

6. Sadeghie Aharai S, Arshi S, Iranparvar M, et al. The effect of complications of type II diabetes on patients' quality of life. Med J Ardabil Univ Med Sci. 2009; 8: 394-402.

7. Komaratat C, Auemaneekul N, Kittipichai W. Quality of life for type II diabetes mellitus patients in a suburban tertiary hospital in Thailand. Journal of Health Research. 2020; 35(1): 3-14, doi: 10.1108/jhr-05-2019-0100.

8. Shafiee-Kandjani A, Hosseinpour M, Shoja $H$, et al. Assessing quality of life and general health status in married women with type II diabetes in Tabriz. Depiction Of Health. 2018; 9(3): 195-205.

9. Fang L, Karakiulakis G, Roth M. Are patients with hypertension and diabetes mellitus at increased risk for COVID-19 infection? Lancet Respir Med. 2020; 8(4): e21, doi: 10.1016/S2213-2600(20)301168, indexed in Pubmed: 32171062.

10. lacobellis G. COVID-19 and diabetes: Can DPP4 inhibition play a role? Diabetes Res Clin Pract. 2020; 162: 108125, doi: 10.1016/j. diabres.2020.108125, indexed in Pubmed: 32224164.

11. Gupta R, Ghosh A, Singh AK, et al. Clinical considerations for patients with diabetes in times of COVID-19 epidemic. Diabetes Metab Syndr. 2020; 14(3): 211-212, doi: 10.1016/j. dsx.2020.03.002, indexed in Pubmed: 32172175.

12. Malek M, Hosseinpanah F, Aghaei Meybodi HR, et al. Diabetes management during the COVID-19 pandemic: an iranian expert opinion statement. Arch Iran Med. 2020; 23(8): 564-567, doi: 10.34172/aim.2020.61, indexed in Pubmed: 32894970.

13. Singh AK, Agrawal B, Sharma A, et al. COVID-19: Assessment of knowledge and awareness in Indian society. J Public Aff. 2020 [Epub ahead of print]: e2354, doi: 10.1002/pa.2354, indexed in Pubmed: 32904779.

14. Salari N, Hosseinian-Far A, Jalali R, et al. Prevalence of stress, anxiety, depression among the general population during the COVID-19 pandemic: a systematic review and meta-analysis. Global Health. 2020; 16(1): 57, doi: 10.1186/s12992-02000589-w, indexed in Pubmed: 32631403.

15. Füzéki E, Groneberg DA, Banzer W. Physical activity during COVID-19 induced lockdown: recommendations. J Occup Med Toxicol. 2020; 15: 25, doi: 10.1186/s12995-020-00278-9, indexed in Pubmed: 32817753.

16. Kent EE, Ornstein KA, Dionne-Odom JN. The family caregiving crisis meets an actual pandemic. J Pain Symptom Manage. 2020; 60(1): e66-e69, doi: 10.1016/j.jpainsymman.2020.04.006, indexed in Pubmed: 32283220.

17. Abdi A, Jalilian M, Sarbarzeh PA, et al. Diabetes and COVID-19: A systematic review on the current evidences. Diabetes Res Clin Pract. 2020; 166: 108347, doi: 10.1016/j.diabres.2020.108347, indexed in Pubmed: 32711003.

18. Fisher J, Languilaire JC, Lawthom R, et al. Community, work, and family in times of COVID-19. Community, Work \& Family. 2020; 23(3): 247-252, doi: 10.1080/13668803.2020.1756568. 